\title{
Características clínicas y de laboratorio en pacientes pediátricos ambulatorios con Fiebre Dengue
}

\author{
Clinical and laboratory characteristics in pediatric \\ outpatients with Dengue Fever
}

\author{
Nicolás González Perrota ${ }^{1,2}$, Soraya Araya ${ }^{3}$, Dolores Lovera ${ }^{1}$, Celia Martínez de Cuellar ${ }^{1,2}$, \\ Antonio Arbo Sosa ${ }^{1,2}$
}

\section{RESUMEN}

Introducción: La fiebre dengue (FD) ha representado en nuestro país una de las causas más frecuentes de consulta en la última década. Objetivos: Describir las características clínicas y de laboratorio de los pacientes con dengue observados en la unidad de atención ambulatoria de un centro de referencia. Materiales y Métodos: Se incluyeron todos los casos de dengue en pacientes menores de 20 años asistidos en la unidad de consulta ambulatoria del Instituto de Medicina Tropical, en el período entre noviembre de 2015 y marzo 2016. En función a una base estructurada de colección de datos, se analizaron las características demográficas, clínicas y de laboratorio. Resultados: Se incluyeron 1491 casos de dengue. La edad media fue de $12 \pm 5$ años, correspondiendo 58 casos (4\%) al grupo etario <2 años, 438 (29\%) al grupo $>2-9$ años y $995(67 \%)$ al grupo $>9$ años $(p<0.01)$. La distribución de sexo fue similar (fem/masc 1.04:1). Presentaron fiebre los 1491 pacientes (100\%). La cefalea, mialgias y artralgias se observaron mas frecuentemente en los pacientes $>9$ años, constatándose en el 79\%, 60\% y 55\% de los casos ( $p<0,01$ comprado con otros grupos etarios). En cambio, el exantema fue más frecuente en los menores de 2 años ( $36 \%$ ) ( $p<0,01$ al comparar con los niños mayores de 2 años). Laboratorialmente la frecuencia de leucopenia fue mayor en los mayores de 2 años (72\% en el grupo de 2-9 años y $81 \%$ en los $>9$ años) al compáralos con los menores de 2 años $(p<0,01)$. La detección del antígeno no estructural NS1 del dengue se observó en la mayoría de los casos (84\%). Conclusiones: El grupo etario predominante que consulta por dengue es el de los niños $>9$ años. Se identificaron variables clínicas (exantema más frecuente en lactantes y algias en niños $>9$ años) y laboratoriales

\section{ABSTRACT}

Introduction: Dengue fever (DF) represents one of the most frequent causes of consultation during the last decade in our country. Objectives: To describe the clinical and laboratory characteristics of dengue patients presenting to the ambulatory care unit of a pediatric reference center. Materials and Methods: We included all cases of dengue in patients under 20 years of age who presented to the outpatient consultation unit of the Institute of Tropical Medicine between November 2015 and March 2016. Based on a structured collection of data, we analyzed their demographic, clinical and laboratory characteristics. Results: 1491 cases of dengue were included. The mean age was $12 \pm 5$ years, 58 cases $(4 \%)$ were in patients aged $<2$ years, $438(29 \%)$ aged $>2-9$ years and $995(67 \%)$ aged $>9$ years $(\mathrm{p}<0.01)$. The sex distribution was similar (fem / masc 1.04: 1). The 1491 patients all had fever $(100 \%)$. Headache, myalgias and arthralgias were observed more frequently in patients $>9$ years old, reported in $79 \%, 60 \%$ and $55 \%$, respectively, of cases (p $<0.01$ compared to other age groups). In contrast, a rash was more frequent in children under 2 years $(36 \%)(\mathrm{p}<0.01$ when compared with children over 2 years). Laboratorially, the frequency of leukopenia was higher in those older than 2 years (72\% in the group of 2-9 years and $81 \%$ in those $>9$ years) when compared with those under 2 years ( $\mathrm{p}<0.01)$. Detection of non-structural dengue NS1 antigen was observed in the majority of cases $(84 \%)$. Conclusions: The predominant age group that consults for dengue is that of children $>9$ years. Clinical variables (rashes in infants and algias in children $>9$ years old) and laboratory variables (rare cytopenias in infants) were identified depending on the age group. The sensitivity of

\footnotetext{
${ }^{1}$ Ministerio de Salud Pública y Bienestar Social, Instituto de Medicina Tropical. Asunción, Paraguay.

${ }^{2}$ Universidad Nacional de Asunción, Facultad de Ciencias Médicas. San Lorenzo, Paraguay.

${ }^{3}$ Ministerio de Salud Pública y Bienestar Social, Programa de Enfermedades Inmunoprevenibles. Asunción, Paraguay. Correspondencia: Dr. Antonio Arbo Correo: antonioarbo@hotmail.com Conflicto de interés: Los autores declaran no poseer conflicto de interés Recibido: 22/11/2019 Aceptado:5/02/2020 DOI: https://doi.org/10.31698/ped.47012020002
} 
(citopenias poco frecuentes en lactantes) que dependen del grupo etario. La sensibilidad de la antigenemia ha sido excelente y similar en los grupos etarios.

Palabras clave: Dengue, Pediatría, atención ambulatoria. antigenemia testing was excellent and similar in all age groups.

Keywords: Dengue, Pediatrics, outpatient care.

\section{INTRODUCCIÓN}

El dengue (FD) es una infección aguda causada por el virus del dengue (DENV), transmitida principalmente por el Aedes aegypti y representa un problema de importante impacto en salud pública en los países tropicales y subtropicales ${ }^{(1)}$.

El DENV corresponde al género Flavivirus de la familia Flaviviridae, con cuatro serotipos filogenética y antigénicamente distintos (DENV-1, DENV-2, DENV-3 y DENV-4) $)^{(2,3)}$. La infección por DENV tiene un amplio espectro de presentaciones clínicas pudiendo causar desde infección subclínica a formas graves con choque y complicaciones (hemorragias, hepatitis, miocarditis, encefalitis) $^{(4)}$.

Las manifestaciones clínicas del dengue incluyen comúnmente fiebre, erupción cutánea, síntomas hemorrágicos leves, dolor de cabeza, dolor ocular, artralgia, mialgia, náuseas y vómitos. En los casos habituales evoluciona sin complicaciones, durando en general el cuadro 3 a 7 días tras los cual hay una recuperación sin complicaciones, siendo el manejo clínico totalmente ambulatorio ${ }^{(5,6)}$. En algunos casos sin embargo el dengue evoluciona a formas severas que incluso ponen en riesgo la vida.

Las investigaciones realizadas tanto en el país como a nivel internacional han sido principalmente enfocadas en pacientes hospitalizados ${ }^{(7,8)}$. De ahí el objetivo del presente estudio fue describir las características clínicas y laboratoriales de los pacientes con dengue que no han requerido hospitalización.

\section{MATERIALES Y MÉTODOS}

Diseño del estudio. El presente estudio ha sido observacional, descriptivo retrospectivo, transversal con componente analítico, basado en la revisión de historias clínicas de los pacientes que consultaron al Departamento de Urgencias del Servicio de Pediatría del Instituto de Medicina Tropical (IMT) entre noviembre de 2015 y marzo de 2016. El IMT es el principal centro de referencia para enfermedades infecciosas y tropicales en Paraguay.

Periodo de estudio y población enfocada. Se incluyeron pacientes de 1 mes a 20 años de vida, que consultaron por fiebre más dos de los siguientes síntomas: mialgias, artralgias, cefalea, algias (dolor retroocular, mialgias, artralgias, dolor abdominal), exantema macular o maculopapular, y/o vómitos. El diagnóstico confirmatorio de dengue se basó en la presencia de una prueba positiva de RT-PCR o antigenemia NS1 positiva, o detección de novo de IgM. El diagnóstico igualmente fue confirmado en caso de presencia de un nexo epidemiológico familiar. Pacientes en quienes los datos del registro clínico fueron insuficientes fueron excluidos del estudio.

Definiciones. Para los propósitos del presente estudio se establecieron las siguientes definiciones:

Fiebre Dengue: infección viral aguda que se manifiesta clínicamente por fiebre más dos de los siguientes síntomas: mialgias, artralgias, cefalea, dolor retro-ocular, exantema macular o maculopapular, vómitos y dolor abdominal; confirmado en caso de PCR positiva para el virus del dengue, presencia del antígeno NS1, serología positiva, o nexo epidemiológico.

Fiebre Dengue con signos de alarma: cuadro febril característico más uno de los siguientes: dolor abdominal intenso o abdomen doloroso a la palpación, vómitos persistentes, acumulación clínica de líquidos, sangrado de mucosas, letargia o agitación, hepatomegalia $>2 \mathrm{~cm}$, laboratorio con aumento del hematocrito concurrente con rápida disminución del número de plaquetas. 
Dengue grave: cuadro febril característico, con signos de alarma, extravasación del plasma (signos de shock por dengue y serositis), hemorragia grave y/o compromiso grave de órganos (incremento en los niveles de enzimas hepáticas ALT (GPT) y AST (GOT) >1000 UI, alteración de la conciencia, insuficiencia renal, insuficiencia cardiaca, miocarditis).

Síndrome de Choque por Dengue: Fiebre Dengue con disminución de la perfusión tisular con o sin hipotensión, afectación del sensorio y oligoanuria.

Recolección de datos. El muestreo fue no probabilístico de casos consecutivos. Las variables demográficas estudiadas fueron edad, sexo y procedencia. Se revisaron los siguientes datos clínicos: presencia de fiebre, mialgias, artralgias, cefalea, exantema, vómitos, dolor abdominal, signos de mala perfusión periférica (llenado capilar menor a 2 segundos). No se incluyó al grupo menor de 2 años en el análisis de los síntomas subjetivos (cefalea, mialgias y artralgias). El perfil de laboratorio estudiado fue la determinación de leucocitos, hematocrito (Hto) y plaquetas, y el resultado de la prueba rápida NS1/IgM/IgG por inmunocromatografía.

Análisis Estadístico. Para la descripción y el análisis se dividieron los grupos de estudio según el grupo etario en: $<2$ años, de 2 a 9 años y $>9$ años. Los datos se ingresaron a una planilla electrónica y posteriormente analizados con el paquete estadístico Epi-Info versión 7. Se utilizaron medidas descriptivas de tendencia central y de dispersión. Para el análisis de variables cualitativas se utilizó la prueba de $X^{2}$ y el test de Fisher según necesidad, y la prueba $T$ Student para variables cuantitativas de distribución normal. Se consideró el nivel de significancia el valor de $p<0,05$.

Aspectos éticos. El estudio fue aprobado por el comité de ética del Instituto de Medicina Tropical.

\section{RESULTADOS}

Datos Demográficos. En el periodo de estudio fueron evaluados 1491 pacientes que consultaron en el Departamento de Urgencias del Instituto de
Medicina Tropical que reunieron los criterios de inclusión. Los datos demográficos de la población general se detallan en la Tabla 1. El promedio de edad fue de $12 \pm 5$ años, la relación sexo femenino/masculino fue similar, con una proporción 1,05:1 y la población mayor de 9 años fue significativamente mayor que los menores de 9 años $(p<0.05)$.

Tabla 1. Datos demográficos y presentación clínica de pacientes con Fiebre Dengue.

\begin{tabular}{lcc}
\hline & $\mathbf{n}=\mathbf{1 4 9 1}$ & $\%$ \\
\hline Edad & 58 & 4 \\
$<2$ años & 438 & 29 \\
$2-9$ años & 995 & 67 \\
$>9$ años & & \\
Sexo & 724 & 49 \\
Masculino & 767 & 51 \\
Femenino & & \\
Procedencia & 1081 & 73 \\
Asunción & 388 & 26 \\
Central & 21 & 1 \\
Otros & & \\
\hline
\end{tabular}

Características Clínicas. Los 1491 (100\%) pacientes presentaron fiebre. Se observó cefalea en 1038 casos (70\%), mialgias en $845(57 \%)$ y artralgias en 662 pacientes $(44 \%)$. Con menos frecuencia, 366 pacientes (25\%) presentaron vómitos, $237(16 \%)$ dolor retroocular, $299(20 \%)$ dolor abdominal, 216 (14\%) exantema y 216 casos (14\%) sangrado leve.

El análisis según los grupos etarios se describe en la Tabla 2.

Datos de Laboratorio. Se dispusieron análisis de laboratorio en 806 pacientes. La biometría hemática mostró un recuento medio de leucocitos sanguíneos de $4679 \pm 2534 / \mathrm{mm} 3$, un valor del hematocrito $39 \pm 4 \%$ y un recuento medio de plaquetas de $187073 \pm 59607 / \mathrm{mm} 3$. En relación a estos valores, 612 $(76 \%)$ pacientes exhibieron leucocitos $<5000 / \mathrm{mm} 3,64$ (8\%) hematocrito $>45 \%$ y $333(41 \%)$ plaquetas $<150000 / \mathrm{mm} 3$. La Tabla 3 muestra los valores comparativos de la biometría hemática según los grupos etarios. Solo un paciente del grupo etario menor de 9 años presentó hemoconcentración, siendo esta figura en los mayores de 9 años del $12 \%(p<0.05)$.

Antígenemia y serología. Estudios laboratoriales confirmatorios de dengue se dispusieron en 544 
pacientes. En 456 pacientes (84\%) el antígeno NS1 fue positivo, mientras que la presencia de IgM antidengue fue positiva en 54 pacientes $(7 \%)$. Veintisiete pacientes (3\%) presentaron al ingreso niveles de IgG positiva anti-dengue. La Tabla 4 muestra el análisis por grupo etario.

Tabla 2. Presentación clínica según grupo etario en pacientes con Fiebre Dengue.

\begin{tabular}{lccccc}
\hline & $\begin{array}{c}<\mathbf{2 a n ̃ o s} \\
(\mathbf{n = 5 8})\end{array}$ & $\begin{array}{c}\mathbf{2 - 9} \text { años } \\
(\mathbf{n = 4 3 8 )}\end{array}$ & $\begin{array}{c}>\mathbf{9} \text { años } \\
(\mathbf{n = 9 9 5 )}\end{array}$ & $\begin{array}{c}\boldsymbol{P} \\
\text { OR } \\
\text { (IC 95\%) }\end{array}$ \\
\hline Fiebre & $58(100 \%)$ & $438(100 \%)$ & $995(100 \%)$ & 1 & \\
Cefalea & No Valorable & $247(56 \%)$ & $791(79 \%)$ & $<0,0001^{*}$ & $0.43(0,33-0,55)$ \\
Mialgias & No Valorable & $162(37 \%)$ & $683(69 \%)$ & $<0,0001^{*}$ & $0,26(0,21-0,33)$ \\
Artralgias & No Valorable & $109(25 \%)$ & $553(55 \%)$ & $<0,0001^{*}$ & $0,26(0,20-0,34)$ \\
Vómitos & $12(21 \%)$ & $120(27 \%)$ & $234(23 \%)$ & $0,58^{*}$ & $0,7(0,4-1,5)$ \\
Dolor Abdominal & No Valorable & $97(22 \%)$ & $201(20 \%)$ & $0,44^{*}$ & $1(0,8-1,5)$ \\
Exantema & $21(36 \%)$ & $72(16 \%)$ & $123(12 \%)$ & $<0,001^{*}$ & $1,6(1,2-2,2)$ \\
\hline
\end{tabular}

Tabla 3. Datos de Hemograma según grupo etario en pacientes con Fiebre Dengue.

\begin{tabular}{lcccc}
\hline & $\begin{array}{c}<\text { 2años } \\
(\mathbf{n}=\mathbf{3 5})\end{array}$ & $\begin{array}{c}\mathbf{2 - 9} \text { años } \\
(\mathbf{n}=\mathbf{2 3 7})\end{array}$ & $\begin{array}{c}>\mathbf{9} \text { años } \\
(\mathbf{n}=\mathbf{5 3 4})\end{array}$ & $\boldsymbol{P}$ \\
\hline Hematocrito & $34 \pm 4 \%$ & $37 \pm 3 \%$ & $40 \pm 4 \%$ & $\mathrm{~N} S$ \\
Plaquetas & $223424 \pm 73374$ & $195551 \pm 63769$ & $168470 \pm 57081^{*}$ & $<0,01$ \\
Leucopenia & $9(26 \%)$ & $170(72 \%)^{*}$ & $433(81 \%)^{*}$ & $<0,01$ \\
Hematocrito $>\mathbf{4 5} \%$ & $1(3 \%)$ & $0(0 \%)$ & $63(12 \%)^{* * *}$ & $\mathrm{NS}$ \\
Trombocitopenia & $4(11 \%)$ & $73(31 \%)^{*}$ & $256(48 \%)^{*}$ & $<0,01$ \\
\hline
\end{tabular}

*T Student $\quad * *=x 2 \quad \mathrm{y} * * *$ Test de Fisher

Tabla 4. Serología y Antigenemia según grupo etario en pacientes con Fiebre Dengue.

\begin{tabular}{cccccc}
\hline & $\begin{array}{c}<\text { 2años } \\
(\mathbf{n}=\mathbf{2 7})\end{array}$ & $\begin{array}{c}\mathbf{2 - 9} \text { años } \\
(\mathbf{n = 1 7 0 )}\end{array}$ & $\begin{array}{c}>\mathbf{9} \text { años } \\
(\mathbf{n}=\mathbf{3 4 7})\end{array}$ & $\boldsymbol{P}$ & $\begin{array}{c}\text { OR } \\
(\text { IC 95\%) }\end{array}$ \\
\hline Ns1 & $22(81 \%)$ & $150(88 \%)$ & $281(81 \%)$ & NS & \\
$\lg M$ & $7(26 \%)^{*}$ & $14(8 \%)$ & $32(9 \%)$ & $<0,05$ & $0,2(0,1-0,6)$ \\
$\operatorname{lgG}$ & $1(4 \%)$ & $8(5 \%)$ & $18(5 \%)$ & $\mathrm{NS}$ & \\
\hline
\end{tabular}

\section{DISCUSIÓN}

El presente estudio presenta una descripción detallada de las manifestaciones clínicas de la infección por el virus del dengue en niños y adolescentes que consultaron, pero no se hospitalizaron (por no reunir criterios) en un hospital de referencia de enfermedades infecciosas del Paraguay. El perfil demográfico encontrado es similar al reportado en otros países ${ }^{(9,10)}$. En la experiencia de de Souza y $\mathrm{col}^{(10)}$ en Rio Janeiro solo una minoría de casos se observó en menores de 1 año, concentrándose los casos en edades mayores, mas importantemente en los grupos etarios mayores de 9 años.
En nuestra serie la proporción de pacientes de sexo masculino y femenino ha sido similar. Sin embargo reportes de otros países muestran datos contradictorios al respecto. Así, mientras en el estudio de de Souza, et al. ${ }^{(10)}$ se encontró la misma proporción, Hammond NS y col $^{(11)}$ en Nicaragua, y Armien y col ${ }^{(9)}$ reportan mayor número de casos en el sexo femenino; en cambio Nagaram y col ${ }^{(12)}$ y Dangi y $\operatorname{col}^{(13)}$ lo señalan predominantemente en el sexo masculino.

El hecho que la mayoría de los casos han procedido de la ciudad capital y ciudades vecinas confirma la observación que el dengue es una enfermedad que 
predomina en áreas urbanas. Diferentes reportes de áreas geográficas diversas confirman esta observación $^{(14,15)}$. El hallazgo de signos y síntomas inespecíficos en el presente estudio, es similar a reportes de otros autores ${ }^{(16)}$. La comparación de la presentación clínica según los grupos etarios, mostró un predominio de cefalea, mialgias y artralgias en los mayores de 9 años y de exantema en los lactantes. Publicaciones similares han reportado la misma distribución en la presentación clínica ${ }^{(10,13)}$. En la publicación de Kittigul et al. ${ }^{(17)}$, que compara la presentación clínica de adultos y niños, se observa también el predominio de las algias en los adultos. La aparición de exantema fue más frecuente en los lactantes en comparación con los niños mayores. La mayor frecuencia de exantemas en los lactantes fue reportada por otros autores ${ }^{(11)}$.

En el análisis de los datos de laboratorio se encontró mayor frecuencia de leucopenia y trombocitopenia en los pacientes mayores de 2 años. Son escasos los trabajos que analizaron el perfil biométrico en casos de dengue que no se hospitalizan ${ }^{(18)}$. Sin embargo, el comportamiento del recuento de glóbulo blancos, y plaquetas muestra similar perfil a los reportado en ellos.

En la presente serie la demostración del antígeno proteico no estructural NS1 mostró alta sensibilidad, hallándose positiva en el $83 \%$ de los casos, similar a lo reportado en otras series ${ }^{(19,20)}$. Solo una minoría exhibió niveles de $\operatorname{IgM}$ positivos, posiblemente porque la prueba se determinó en general dentro de los primeros cinco días de la enfermedad, tiempo en el cual la misma es habitualmente negativa.

El presente estudio presenta las limitaciones propias de las revisiones retrospectivas. Cuatro de cada 10 pacientes no tuvieron determinación del hemograma y 6 de 10 pacientes no tuvieron confirmación diagnostica por antigenemia o serología. Sin embargo, revela el perfil demográfico de los pacientes con dengue que consultan a un hospital de referencia en el Paraguay, mostrando el predominio etario y la variabilidad de la sintomatología clínica relacionada al mismo. Confirma además la buena sensibilidad de la determinación del antígeno no estructural NS1 en el diagnóstico del dengue.

\section{CONCLUSIONES}

Hubo predominio de pacientes mayores de 9 años con Dengue en la consulta ambulatoria quienes presentaron predominio de síntomas subjetivos y menos exantema que los grupos etarios menores. En el aspecto laboratorial el contaje de leucocitos y plaquetas fue significativamente inferior que el grupo de niños de menor edad.

\section{REFERENCIAS}

1. Halstead SB. Dengue Virus-Mosquito Interactions. Annu Rev Entomol. 2008; 53:273-291.

2. Waman VP, Kolekar P, Ramtirthkar MR, Kale MM, Kulkarni-Kale U. Analysis of genotype diversity and evolution of Dengue virus serotype 2 using complete genomes. Peer J. 2016; 4:e2326. DOI: 10.7717/peerj.7852.

3. Yamashita A, Sasaki T, Kurosu T, Yasunaga T, Ikuta K. Origin and distribution of divergent dengue virus: Novel database construction and phylogenetic analyses. Future Virol. 2013; 8:1061-1083.

4. World Health Organization. Dengue: Guidelines for Diagnosis, Treatment, Prevention and Control. New ed. Geneva. Switzerland: World Health Organization; 2009.
5. Halstead SB. Dengue. Lancet. 2007;370(9599):1644-1652.

6. Simmons CP, Farrar JJ, Nguyen van VC, Wills B. Dengue. N Engl J Med. 2012;366:1423-1432.

7. Lovera D, Araya S, Mesquita MJ, Avalos C, Ledesma S, Arbo A. Prospective applicability study of the new dengue classification system for clinical management in children. Pediatr Infect Dis J. 2014; 33:933-935.

8. Lovera D, Martinez de Cuellar C, Araya S, Amarilla S, Gonzalez N, Aguiar C, et al. Clinical characteristics and risk factors of dengue shock syndrome in children. Pediatr Infect Dis J. 2016;35:1294-1299. 
9. Armien B, Suaya JA, Quiroz E, Sah BK, Bayard V, Marchena L, et al. Clinical characteristics and national economic cost of the 2005 dengue epidemic in Panama. Am J Trop Med Hyg. 2008; 79(3):364-371.

10. Souza LJ, Pessanha LB, Mansur LC, Souza LA, Ribeiro $\mathrm{MB}$, et al. Comparison of clinical and laboratory characteristics between children and adults with dengue. Brazilian J Infect Dis. 2013; 17(1):27-31.

11. Hammond SN, Balmaseda A, Pérez L, Tellez Y, Saborío SI, Mercado JC, et al. Differences in dengue severity in infants, children, and adults in a 3-year hospital-based study in Nicaragua. Am J Trop Med Hyg. 2005; 73(6):1063-70.

12. Nagaram PP, Piduru P, Munagala VK, Matli VV. Clinical and laboratory profile and outcome of dengue cases among children attending a tertiary care hospital of South India. Int J Contemp Pediatr. 2017; 4:1074. DOI: 10.18203/2349-3291.ijcp20171731.

13. Dangi V, Singh P. Clinical epidemiological profile of patients in tertiary care center Hamidia Hospital Bhopal Madhya Pradesh. Int J Sci Res. 2018;7(3):25-26.

14. Udayanga L, Gunathilaka N, Iqbal MCM, Lakmal K, Amarasinghe US, Abeyewickreme W. Comprehensive evaluation of demographic, socio-economic and other associated risk factors affecting the occurrence of dengue incidence among Colombo and Kandy Districts of Sri Lanka: A cross-sectional study. Parasites and Vectors. 2018; 11:1-18.
15. Bhatt S, Gething PW, Brady OJ, Messina JP, Farlow AW, Moyes CL, et al. The global distribution and burden of dengue. Nature 2013; 496:504-507.

16. Potts JA, Rothman AL. Clinical and laboratory features that distinguish dengue from other febrile illnesses in endemic populations. Trop Med Int Health. 2008; 13(11):1328-1340.

17. Kittigul L, Pitakarnjanakul P, Sujirarat D, Siripanichgon $\mathrm{K}$. The differences of clinical manifestations and laboratory findings in children and adults with dengue virus infection. J Clin Virol. 2007; 39:76-81.

18. Trung DT, Thao le TT, Dung NM, Ngoc TV, Hien TT, Chau NV, et al. Clinical features of dengue in a large Vietnamese cohort: intrinsically lower platelet counts and greater risk for bleeding in adults than children. PLoS Negl Trop Dis. 2012;6(6):e1679. DOI: 10.1371/journal.pntd.0001679

19. Dussart P, Petit L, Labeau B, Bremand L, Leduc A, Moua D, et al. Evaluation of two new commercial tests for the diagnosis of acute dengue virus infection using NS1 antigen detection in human serum. PLoS Negl Trop Dis. 2008 Aug 20;2(8):e280. DOI: 10.1371/journal.pntd.0000280.

20. Shih HI, Hsu HC, Wu CJ, Lin CH, Chang CM, Tu YF, et al. Applications of a rapid and sensitive dengue DUO rapid immunochromatographic test kit as a diagnostic strategy during a dengue type 2 epidemic in an Urban City. PLoS One. 2016; 11:e0158437. DOI: 10.1371/journal.pone.0158437. 